\title{
Hard X-ray Microscopy with Elemental, Chemical, and Structural Contrast
}

\author{
C.G. Schroer ${ }^{a}$, P. Boye ${ }^{a}$, J.M. Feldkamp ${ }^{a}$, J. Patommel ${ }^{a}$, A. Schropp ${ }^{a}$, D. Samberg ${ }^{a}$,

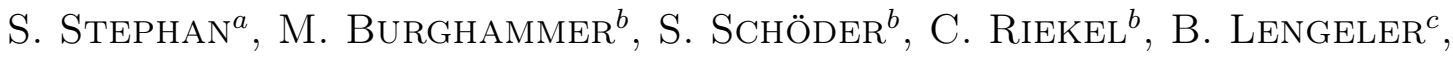 \\ G. Falkenberg ${ }^{d}$, G. Wellenreuther ${ }^{d}$, M. Kuhlmann ${ }^{e}$, R. Frahm $^{f}$, \\ D. LÜTZENKIRCHEN-HECHT ${ }^{f}$ AND W.H. SCHROEDER ${ }^{g}$ \\ ${ }^{a}$ Institute of Structural Physics, Technische Universität Dresden, D-01062 Dresden, Germany \\ ${ }^{b}$ ESRF, B.P. 220, F-38043 Grenoble, France \\ ${ }^{c}$ II. Physikalisches Institut, RWTH Aachen University, D-52056 Aachen, Germany \\ ${ }^{d}$ HASYLAB at DESY, Notkestr. 85, D-22607 Hamburg, Germany \\ ${ }^{e}$ CELLS ALBA, Cerdanyola del Vallès, Barcelona, Spain \\ ${ }^{f}$ Institute of Materials Science and Department of Physics \\ Bergische Universität Wuppertal, Gauss Str. 20, D-42097 Wuppertal, Germany \\ ${ }^{g}$ ICG-3, Forschungszentrum Jülich GmbH, D-52425 Jülich Germany
}

\begin{abstract}
We review hard X-ray microscopy techniques with a focus on scanning microscopy with synchrotron radiation. Its strength compared to other microscopies is the large penetration depth of hard $\mathrm{x}$ rays in matter that allows one to investigate the interior of an object without destructive sample preparation. In combination with tomography, local information from inside of a specimen can be obtained, even from inside special non-ambient sample environments. Different X-ray analytical techniques can be used to produce contrast, such as X-ray absorption, fluorescence, and diffraction, to yield chemical, elemental, and structural information about the sample, respectively. This makes X-ray microscopy attractive to many fields of science, ranging from physics and chemistry to materials, geo-, and environmental science, biomedicine, and nanotechnology. Our scanning microscope based on nanofocusing refractive X-ray lenses has a routine spatial resolution of about $100 \mathrm{~nm}$ and supports the contrast mechanisms mentioned above. In combination with coherent X-ray diffraction imaging, the spatial resolution can be improved to the $10 \mathrm{~nm}$ range. The current state-of-the-art of this technique is illustrated by several examples, and future prospects of the technique are given.
\end{abstract}

PACS numbers: 68.37.Yz, 41.40.th

\section{Introduction}

A key strength of hard X-ray microscopy is the large penetration depth of hard $\mathrm{x}$ rays in matter. This allows one to investigate the interior of a sample without destructive sample preparation or to study an object in-situ inside a special sample environment, such as a chemical reactor or a pressure cell. In combination with tomography, it yields three-dimensional information from inside the sample.

In addition, hard $\mathrm{x}$ rays are a very attractive probe as a result of their short wavelength and their energy in the range of electronic transitions of many elements. These properties are exploited in many X-ray analytical techniques, such as absorption and fluorescence spectroscopy and X-ray diffraction. In a hard X-ray microscope, these $\mathrm{X}$-ray analytical techniques can be exploited as contrast, giving structural information down to the atomic scale in the case of X-ray diffraction, insight into the chemical environment of an atomic species in the case of absorption spectroscopy, or the elemental composition by X-ray fluorescence analysis. Together with the large penetration depth, this makes hard X-ray microscopy a valuable tool in many sciences, from physics and chemistry [1, 2], bio-medicine [3, 4], to the materials [5-8], earth and environmental sciences [9-14], and nanotechnology [15].

Compared to microscopy with soft $\mathrm{x}$ rays, hard X-ray microscopy is relatively young $[16,17]$. It evolved during the last two decades triggered by the advent of synchrotron radiation sources of the third generation, such as the European Synchrotron Radiation Facility (ESRF) in Grenoble, France, the Advanced Photon Source (APS) near Chicago in the United States, and SPring-8 in Japan, providing hard $\mathrm{x}$ rays and unprecedented brilliance. Besides the sources, significant developments were 
made in the field of hard X-ray optics, and today hard $\mathrm{X}$-ray microscopes are installed at many synchrotron radiation sources.

There are basically two types of X-ray microscopes that are complementary in many ways: full-field and scanning microscopes. While full-field microscopes allow for fast acquisition of 2D transmission images, scanning microscopes can exploit a larger variety of contrast mechanisms. Since more recently, coherent X-ray diffraction imaging techniques are being developed, having both traits of full-field and scanning microscopy.

In the following Sect. 2, these techniques are reviewed and described in more detail, focusing on scanning microscopy. In section 3 , three different examples are given, illustrating the capabilities of scanning microscopy. In section 4 , we conclude and give a short outlook on a future X-ray scanning microscope that is set up at the synchrotron radiation source PETRA III at DESY in Hamburg.

\section{Hard X-ray microscopy}

\subsection{Full-field microscopy}

Hard X-ray microscopy can be roughly subdivided in full-field and scanning microscopy [16-18]. The simplest approach to full-field microscopy at a synchrotron radiation source is shown in Fig. 1a). Here, the sample is illuminated by nearly parallel synchrotron radiation, and its projection image is recorded at a certain distance $L$ behind the sample by a two-dimensional position-sensitive detector. In this scheme, the resolution is predominantly limited by that of the detector, reaching down to slightly below one micrometer $[19,20]$. By recording a series of such images while rotating the sample over $180^{\circ}$, a tomographic data set is obtained that can be reconstructed to yield the three-dimensional inner structure of the specimen [21-23].

With the detector directly behind the object (small $L$ ), the transmission image is dominated by absorption contrast. In addition, at synchrotron radiation sources of the third generation, the source size is small enough to allow one to observe near-field phase contrast [24-26] that can be varied with the detector distance $L$. From a series of such phase contrast images taken at different distances $L$, the wave field behind the object can be reconstructed. Besides the attenuation, this approach also yields the phase shift induced by refraction inside the sample. In combination with tomography, the threedimensional distribution of the complex index of refraction can be reconstructed $[27,28]$. More recently, another type of phase contrast was introduced based on a grating interferometer placed into the beam between the sample and the detector [29-31].

In combination with absorption spectroscopy, projection imaging can yield valuable information about the distribution of the chemical state of a given element. For this purpose, the X-ray energy is scanned around an absorption edge of an element of interest and at each energy an absorption image of the sample is recorded. From

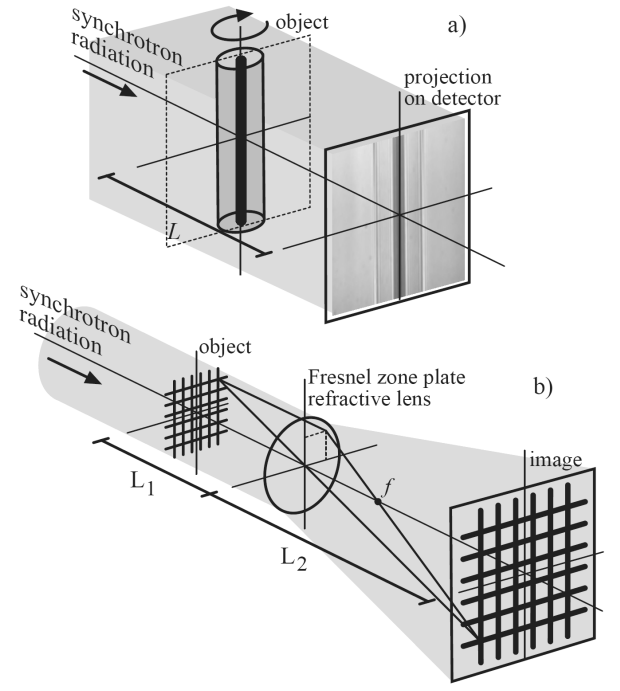

Fig. 1. (a) High-resolution X-ray imaging with the quasi-parallel beam at modern synchrotron radiation sources. (b) Magnified imaging using an X-ray optic, such as parabolic refractive X-ray lenses to generate a magnified image of an object on a two-dimensional position-sensitive X-ray detector. The position of the focus behind the lens is marked by the letter $f$.

these data, the local absorption spectrum can be reconstructed for each pixel [1]. This technique is particularly useful in physical chemistry, e.g., in the study of catalysis, as it allows one to determine in-operando the local oxidation state of a catalyst inside a chemical reactor and learn about catalytic reactions and their kinetics [1, 2, 32-36].

In order to overcome the limitation in the spatial resolution given by the detector, magnifying imaging schemes are required. One way of realizing this is to generate a small secondary X-ray source, for example by focusing the $\mathrm{x}$ rays down to a small spot using an X-ray optic. The sample is then placed in a small distance $L_{1}$ behind the focus and its projection image is recorded on a twodimensional detector located a distance $L_{2}\left(\gg L_{1}\right)$ behind the focus (magnification $L_{2} / L_{1}$ ). A first demonstration of this technique was given using one-dimensional confinement of the X-ray beam by a wave guide [37]. More advanced imaging was recently achieved using a Kirkpatrick-Baez-multilayer optic [38]. The technique always involves phase contrast, and phase retrieval techniques are required to obtain quantitative information about the sample. However, it does not require an X-ray optic behind the object, thus optimally exploiting the $\mathrm{X}$-ray dose applied to the sample.

Alternatively, the sample can be imaged onto the detector by an appropriate X-ray optic, as shown in Fig. 1b). Here, the sample is illuminated by hard $\mathrm{x}$ rays, usually condensed onto the sample by some condenser optic (not shown in Fig. 1). The objective lens is placed in a distance $L_{1}$ behind the sample $\left(L_{1}\right.$ is slightly larger than the focal length $f$ ) and the image is formed a dis- 
tance $L_{2}=L_{1} f /\left(L_{1}-f\right)$ on a two-dimensional positionsensitive detector.

Fresnel zone plates are often used as objective lenses, in particular in the soft X-ray range (cf. for example the many contributions in [16]). Recently, also hard Xray full-field microscopy with a resolution down to $40 \mathrm{~nm}$ was successfully implemented at the APS based on these optics [39]. We have used refractive X-ray lenses as objective lens for magnified imaging and tomography [40, 41]. Resolutions on the scale of $100 \mathrm{~nm}$ have been reached with refractive lenses made of beryllium [42]. More detail about refractive optics is given below in the context of scanning microscopy.

\subsection{Scanning microscopy}

In scanning microscopy, the sample is scanned through a hard X-ray microbeam (cf. Fig. 2). The latter is often generated by imaging the $\mathrm{X}$-ray source onto the sample position in a strongly reducing geometry: the sourceto-optic distance $L_{1}$ is typically much larger than the focal length $f$ of the optic. The image of the source is formed in a distance $L_{2}=L_{1} f /\left(L_{1}-f\right) \gtrsim f$ behind the optic and it is reduced by a factor $L_{1} / L_{2}$. The sample is scanned through the microbeam along the two directions perpendicular to the optical axis. For tomography, the sample is also rotated about the vertical axis (Fig. 2).

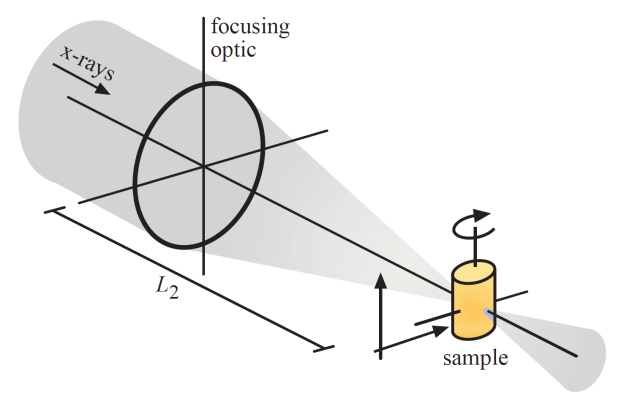

Fig. 2. Schematic diagram of a microbeam-based scanning microscope.

Different contrast mechanisms can be exploited to obtain various pieces of information about the sample. At each position of the scan, the fluorescence, Compton, and elastic scattering signals can be recorded by an energy dispersive detector, the transmission can be measured by a positive-intrinsic-negative (PIN) diode or ionization chamber, or the diffraction from the sample can be registered by a two-dimensional position-sensitive detector. In most cases, the microbeam is monochromatized by a crystal monochromator (relative band width $\left.\Delta E / E \approx 10^{-4}\right)$. However, some diffraction techniques require a white beam (broad spectrum) [43], and for efficient fluorescence mapping the relative band width $\Delta E / E$ can be defined in the percent range by a multilayer optic [44].

As the X-ray beam is confined only in its two lateral dimensions, the measured signal, be it fluorescence, absorption, or diffraction, is obtained from the full thickness of the sample along the beam. If the sample is sufficiently thin, it can be mapped in two translational degrees of freedom perpendicularly to the beam, e.g., [3, 4]. To obtain high spatial resolution also in the third dimension, tomographic scanning techniques need to be used. In order to obtain a single projection for a tomographic slice through the sample, it is first scanned trough the beam in horizontal translation perpendicular to the axis of rotation and the beam direction. At each position of the scan, the signal from the sample is recorded. After completing the projection, the sample is rotated by an integer fraction of $180^{\circ}$ or $360^{\circ}$ and the translational scan is repeated. The procedure ends when a half or full rotation of the sample is completed. To obtain three-dimensional information, a series of parallel slices needs to be acquired [14]. Alternatively, a helical scanning mode can be used [45].

The tomographic reconstruction depends on the contrast chosen. Various reconstruction techniques have been developed for fluorescence [46-52], absorption spectroscopy [53], small-angle X-ray scattering (SAXS) [54, 55], wide angle X-ray powder diffraction [56], and grazing incidence small-angle X-ray scattering (GISAXS) [57].

The spatial resolution of the microscope is limited by the lateral beam size that in turn depends on the $\mathrm{X}$ ray source brilliance and dimensions, the geometry of the setup, and the X-ray optic used to generate it. The requirements in spatial resolution are given by the length scales in the sample. Today, microbeams range from a few tens of micrometers in the laboratory [58] down to a few $10 \mathrm{~nm}$ at modern synchrotron radiation sources. Besides a highly brilliant source, the latter beam sizes require special focusing optics. Over the last two decades, significant advances have been made in the fabrication of $\mathrm{X}$-ray optics. In the soft X-ray range, Fresnel zone plates for spatial resolutions down to $15 \mathrm{~nm}$ have been developed [59]. For hard x rays, total reflection mirrors [60], multilayer mirrors [44], Fresnel zone plates [39, 61], multilayer Laue lenses [62, 63], wave guides [64], and refractive lenses [65] have all been shown to generate foci well below $100 \mathrm{~nm}$. Most of these optics are limited by today's fabrication technology, and there is still room for significant improvements in their numerical aperture [66-69].

Our hard X-ray scanning microscopes are based on refractive X-ray lenses, and all the experiments described in Sect. 3 were carried out with these optics. Therefore, they are briefly reviewed in the following.

For a long time it was thought that refractive lenses could not be realized in the X-ray range, as refraction of hard $\mathrm{x}$-rays in matter is very weak and the attenuation inside all materials is relatively large compared to that of visible light in glass. It was only a decade ago that their feasibility was demonstrated [70]. Since then, there has been a dynamic development, and refractive optics with many different designs were made [71-79]. The main idea followed by all the schemes is to use many refracting surfaces, i.e., focusing the beam by stacking a large number of individual lenses behind each other to form a 
compound lens. For X-ray lenses the choice of material is crucial. The key property of the lens material is minimal attenuation, which requires the use of elements with low atomic number $Z$, such as beryllium $[42,80]$, boron, carbon [81], aluminium, or silicon. Other important issues are the processibility, the stability, and the homogeneity (low scattering) of the lens material [71, 82].

The most important development for X-ray microscopy was that of the rotationally parabolic lens [40, 82]. The rotationally parabolic shape of the lens surface avoids spherical aberrations, allowing for undistorted imaging with these optics. The parabolic shape is the correct aspherical shape in the paraxial approximation, which is fulfilled to high accuracy for all refractive optics. This aspherical shape of the lens is particularly important in the hard X-ray range and opens the way to full-field imaging $[40,41,83]$ and scanning microscopy [80, 84]. Today, these optics are widely used at several synchrotron radiation sources as standard components for beam conditioning and for microbeam production [85].

For refractive X-ray lenses with large apertures, the numerical aperture scales as the inverse square root of the focal length. Therefore, it is advantageous to reduce the focal length of these optics as much as possible to reach a high numerical aperture and thus a high spatial resolution. This argument led to the development of nanofocusing refractive X-ray lenses (NFLs) [86] with particularly short focal distances in the range of one centimeter. The small dimensions of these optics require the use of microfabrication techniques. Currently, they are made out of silicon by optical lithography and deep reactive ion etching. Fig. 3a) shows a scanning electron micrograph of an NFL. NFLs are composed of parabolic cylinder lenses and generate a line focus. In order to obtain a point focus, two such lenses need to be aligned in crossed geometry as shown in Fig. 3b). The two lenses are placed on two separate stages with a total of 10 degrees of freedom. Each lens is separately aligned in the beam with the help of a high resolution X-ray camera. These optics have been demonstrated to generate hard $\mathrm{X}$-ray beams down to about $50 \mathrm{~nm}$ lateral size [65].

In collaboration with beamline ID13 at the ESRF, this optical setup has been included in the new scanning microscope at about $96 \mathrm{~m}$ from the undulator source at the ID13 nanoprobe extension. Fig. 3c) shows the sample region of this scanning microscope, with piezo-mechanical scanning stages for three-dimensional positioning of the sample, an energy dispersive detector, and a beam- defining aperture behind the set of lenses shown in b). Upstream of the lenses, a slit system confines the beam to a rectangular cross section slightly larger than the aperture of the lenses. Below the scanning stage, a rotation stage allows for high precision rotation with minimal wobble and eccentricity. Additional stages for coarse alignment of the sample on the rotation axis above the rotation stage, as well as high precision translations below the rotation stage complete the sample stage. The whole setup is mounted on a specially designed optical
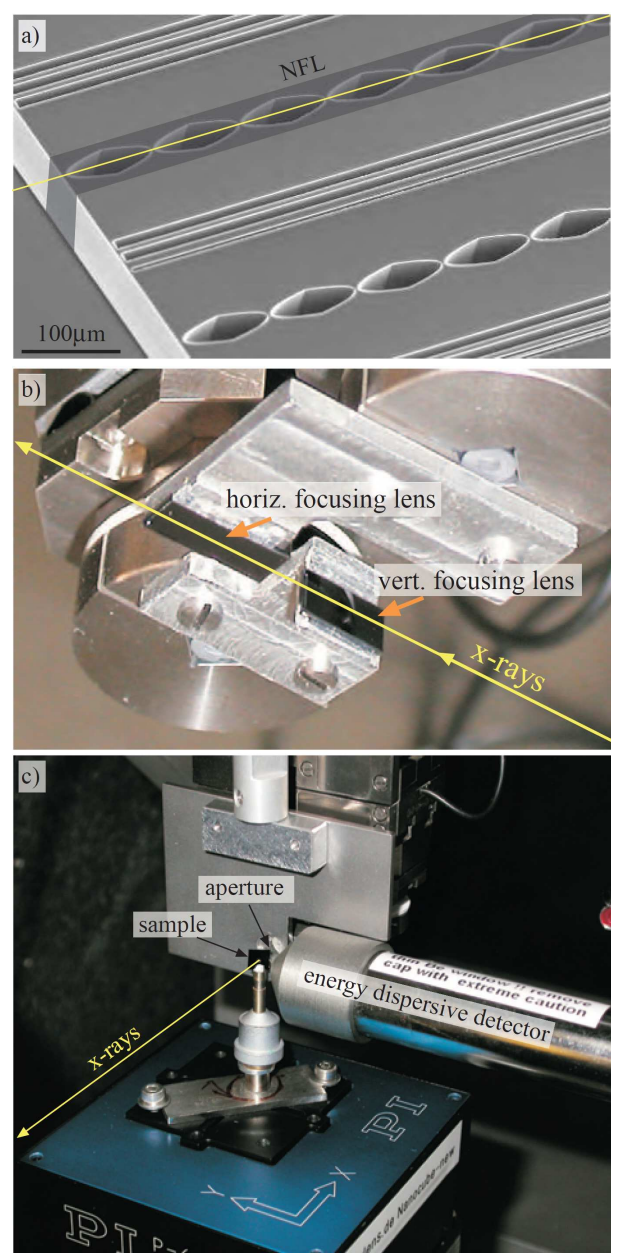

Fig. 3. (a) Nanofocusing refractive X-ray lenses (NFLs). (b) Pair of crossed NFLs pointed to by the arrows and aligned to generate a point focus about $10 \mathrm{~mm}$ behind the horizontally focusing lens. (c) Hard X-ray scanning microscope based on NFLs. The sample is scanned through the beam using a 3D piezo scanner (PI). The rotation for tomographic scans is realized by a rotation stage below the piezo scanner.

table and is kept in a temperature controlled environment $(\Delta T<0.1 \mathrm{~K})$. The beam from the in-vacuum undulator source is monochromatized by a channel cut $\mathrm{Si}$ 111 monochromator, and the lateral coherence length is adapted to the aperture of the NFL optics by appropriate prefocusing with a parabolic refractive X-ray lens made of beryllium located at about $40 \mathrm{~m}$ from the source. The examples given in Sects. 3.1. and 3.3. were obtained with this setup or with a very similar prototype.

\section{Examples}

The following examples illustrate some of the capabilities of X-ray scanning microscopy. While the first two examples show how the element distribution and local zinc speciation can be obtained by scanning microscopy and tomography with fluorescence and absorption spec- 
troscopic contrast, respectively, the last example is more methodological and shows how the spatial resolution can be improved beyond the focus size of the microscope by combining nanofocusing with coherent X-ray diffraction imaging techniques.

\subsection{X-ray Fluorescence mapping and tomography}

For plant physiologists, the distribution of physiologically relevant ions is of great interest to understand transport phenomena inside a plant. Besides their me- chanical function for the defense against pathogens, leaf hairs (trichomes) of plants excrete salts and contribute in this way to the balance of physiologically relevant ions in the plant. To study the latter function, we investigated the element distribution inside a leaf hair of Arabidopsis thaliana by both fluorescence mapping and tomography. Fig. 4a shows the live plant with the trichomes on the leaves. In Fig. 4b, a single trichome is shown in a scanning electron micrograph after freeze drying of a leaf.
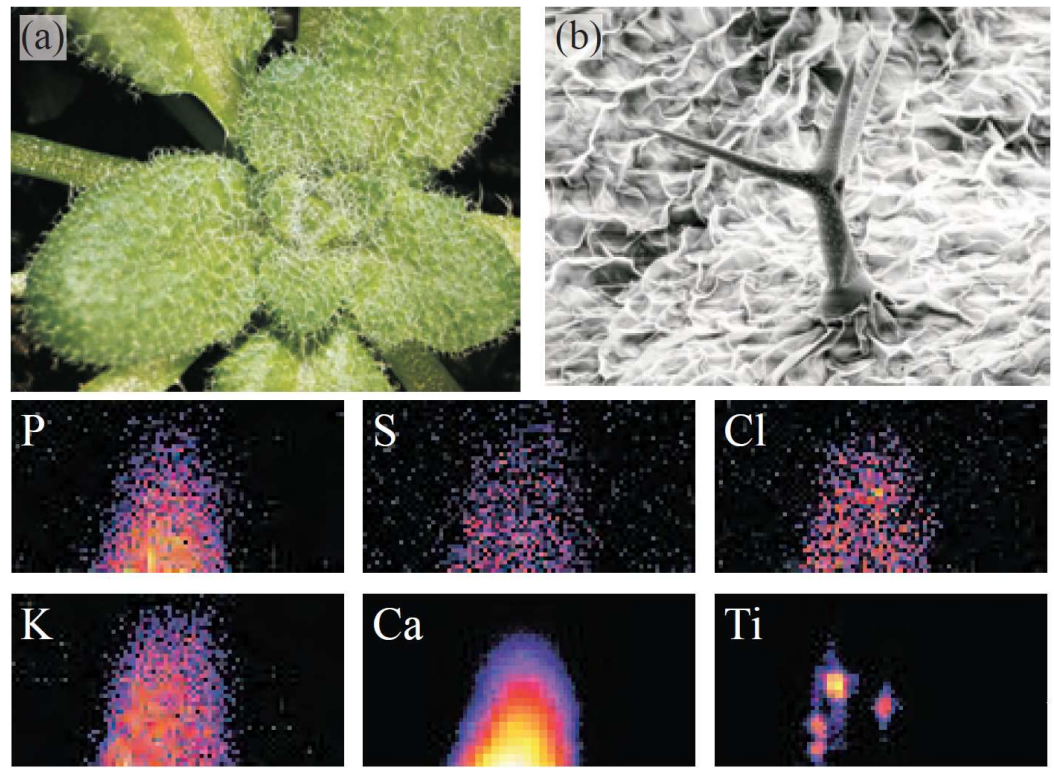

(c)
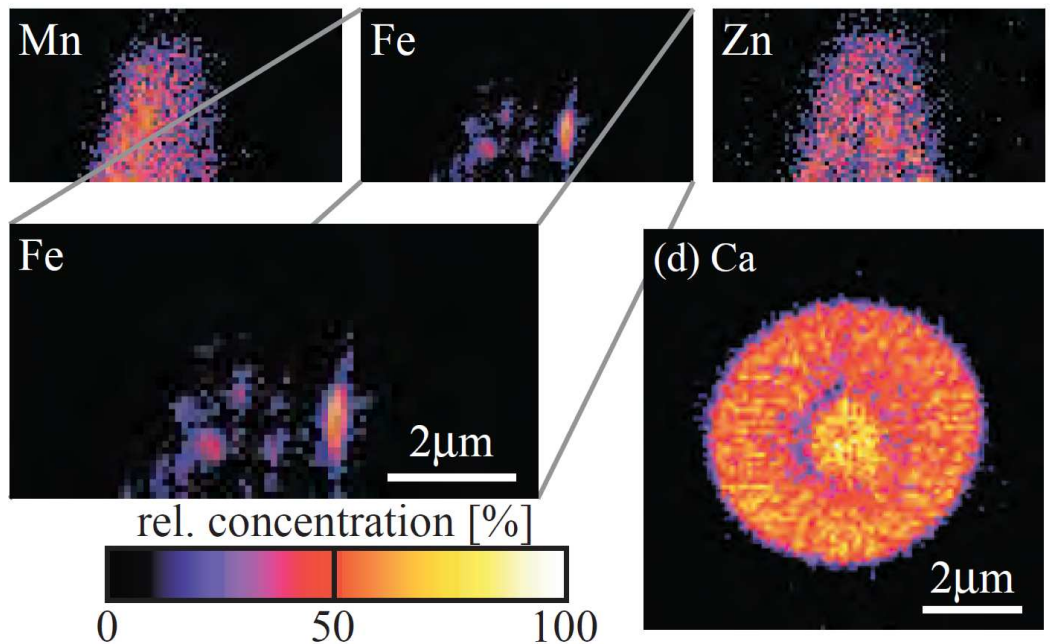

Fig. 4. (a) Photograph of Arabidopsis thaliana with its leaves covered by leaf hairs (trichomes). (b) Scanning electron micrograph of a single trichome on a freeze dried leaf. (c) X-ray fluorescence maps of the tip of a trichome, scanned with a pixel size of $100 \mathrm{~nm}$. (d) Fluorescence tomographic slice (calcium distribution) through a trichome recorded with $100 \mathrm{~nm}$ pixel size. The relative concentration of each elemental species in both parts (c) and (d) is color coded according to the color bar.

A single freeze dried trichome was prepared and scanned at the tip with our scanning microscope at $E=15 \mathrm{keV}$ with a step size of $100 \mathrm{~nm}$ (dwell time $5 \mathrm{~s}$ per point). At each position in the scan, full fluorescence spectra were recorded, from which the distribution of various elements were extracted. Fig. 4c shows the 
two-dimensional maps of these elements. While many of the elements are rather homogeneously distributed about the tip, titanium ( $\mathrm{Ti}$ ) and iron (Fe) show fine structures on on the scale of $100 \mathrm{~nm}$. The physiological reason for this localization is not yet known, but it illustrates the high spatial resolution obtained with our microscope. In addition, the method can provide quantitative element distributions, if the setup is properly calibrated.

The two-dimensional map provides no information on the location of the elements along the beam. For example, it is not possible to determine whether the accumulations of iron and titanium lie inside the trichome or on its periphery. This could be answered by fluorescence tomography.

In another experiment, a fluorescence tomographic scan across a freeze dried trichome near one of the tapered tips was recorded. After fixing the virtual section by adjusting the height of the sample in the beam, it was scanned in horizontal translation in 87 steps of $100 \mathrm{~nm}$, each. At each position of the scan, the full fluorescence spectrum was recorded from the sample in $5 \mathrm{~s}$ of exposure time, exciting the fluorescence with monochromatic radiation $(E=24.3 \mathrm{keV})$. After the translational scan was completed, the sample was rotated by an integer fraction of $180^{\circ}$ and the next projection (translational scan) was recorded. This procedure was repeated until half a rotation of the sample was completed. In the current experiment 60 rotational steps were made with a step size of 3 degrees.

While the integral spectrum of the sample indicates the presence of many elements, the concentrations of elements other than calcium were too low to be reconstructed tomographically in this particular section of the trichome. No accumulations of iron or titanium similar to those shown in Fig. 4c could be found, here. The tomographic reconstruction of the calcium signal was made by filtered backprojection [87], since attenuation effects are negligible for this sample and more complicated reconstruction schemes are not necessary. This is due to the very small size of the sample, its low- $Z$ matrix and the sufficiently high energy of the calcium (Ca) $\mathrm{K} \alpha$ line. The tomographic reconstruction of the Ca distribution is shown in Fig. 4d. The pixel size in the reconstruction is $100 \mathrm{~nm}$. The section of the trichome has a diameter of about $6 \mu \mathrm{m}$, which is significantly smaller than in previous fluorescence tomograms, where attenuation effects were significant $[48,88,89]$.

\subsection{Combining absorption spectroscopy with tomographic scanning microscopy}

$\mathrm{X}$-ray absorption spectroscopy yields valuable information about the chemistry and the local environment of an elemental species. In a typical absorption measurement, the intensity in front of and behind a homogeneous specimen is measured as a function of X-ray energy. When crossing an absorption edge of a given element, the absorption in the sample increases in a characteristic way. Depending on the oxidation state, the absorption edge of the given element is shifted by a few $\mathrm{eV}$. The exact shape of the edge and the near-edge region, extending to about $100 \mathrm{eV}$ above the absorption edge, is called the "X-ray absorption near-edge structure" (XANES) and yields the local projected density of free electronic states around the atom. The extended X-ray absorption fine structure (EXAFS) that extends to about $1 \mathrm{keV}$ above the edge gives the local environment around the atom, e.g., the distances and the number of neighboring atoms.

In practice, many samples are inhomogeneous and their properties change over macroscopic length scales. In this case, absorption spectroscopy can be combined with scanning microscopy or tomography to obtain local information about the sample. To combine these two techniques, the absorption as a function of energy has to be recorded at each position of a scan. As a scanning micrograph typically contains many thousand scan points, efficient acquisition of absorption spectra is crucial. This is possible with a fast scanning monochromator and data acquisition system developed at the University of Wuppertal [90, 91].

We have demonstrated the feasibility of XANES tomography investigating a $\mathrm{Cu} / \mathrm{Zn}$ oxide catalyst inside a reactor capillary $[53,92]$. There, the XANES spectra of different oxidation states of $\mathrm{Cu}$ and $\mathrm{Zn}$ were used as a fingerprint for these different chemical species. They were fitted to the reconstructed spectra to obtain their distribution. Here, we would like to illustrate the method on a biological sample, reconstructing the XANES of zinc in a mycorrhyzal root of a tomato plant. The experiment was carried out at beamline 1-ID at the Advanced Photon Source (APS). Compared to highly concentrated catalyst samples, physiologically relevant concentrations of heavy metals in plants are rather low, making the absorption measurements very challenging.

To acquire a zinc XANES tomogram of the freeze dried tomato root on a cross section, we aligned the cylindrical root with its axis along the vertical rotation axis, chose an appropriate vertical position for the slice and recorded 102 projections over $360^{\circ}$ with 87 steps of $10 \mu \mathrm{m}$ each. The X-ray beam from the fast scanning monochromator was focused to about $10 \mu \mathrm{m}$ using parabolic refractive $\mathrm{X}$ ray lenses made of beryllium. This spatial resolution is appropriate to resolve structures on the tissue level. At each position of the scan, 10 full XANES spectra about the zinc edge $(E=9659 \mathrm{eV})$ were acquired in 1 second, recording the absorption and the fluorescence with a sampling rate of $100 \mathrm{kHz}$. The data at each point were calibrated in energy and summed up.

The transmission and the fluroescence signal were reconstructed using filtered backprojection separately for each energy in the absorption spectrum. In this way, at each location in the reconstruction, a full absorption spectrum was obtained. Attenuation effects of the fluorescence signal are negligible in this case, as the freeze dried sample of about $700 \mu \mathrm{m}$ diameter is made of a low-density low-Z matrix that is transparent to good approximation to the zinc K-fluorescence radiation. Fig. 5a 
shows the attenuation coefficient above the absorption edge on the virtual slice through the sample. Fig. 5b shows the reconstructed fluorescence signal above the absorption edge that is dominated by zinc fluorescence, thus representing to good approximation the zinc distribution on the virtual slice.

\section{(a) absorption}
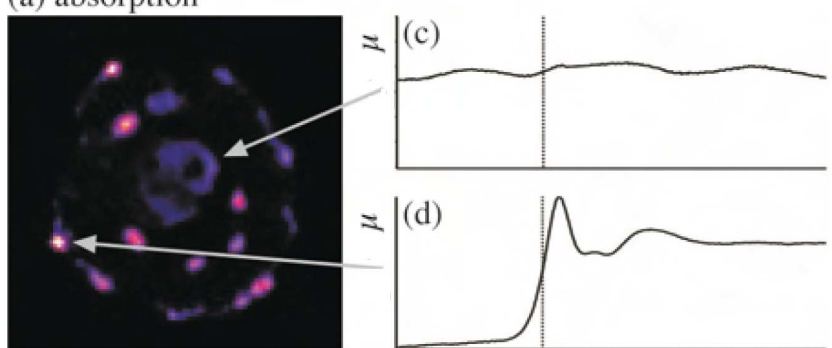

(b) fluorescence
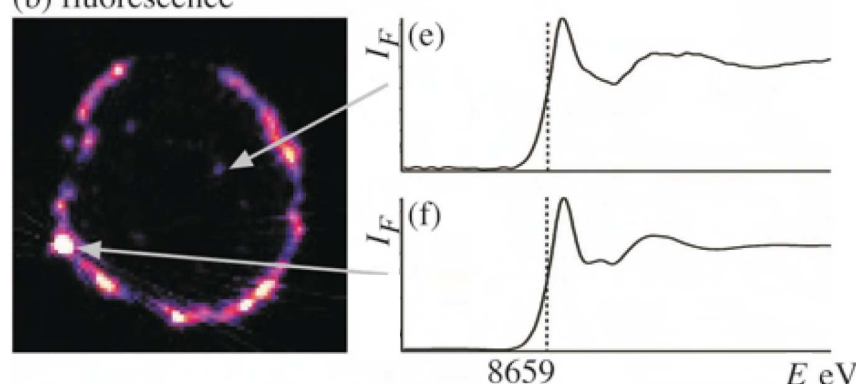

$E \mathrm{eV}$

Fig. 5. (a) Reconstructed absorption across a shock frozen and freeze dried root of a tomato plant $(E \approx$ $9700 \mathrm{eV}$ ) with two reconstructed spectra (c) and (d) at different locations pointed to by the arrows. (b) Reconstructed fluorescence signal recorded in parallel to the attenuation shown in (a), (e) and (f) are absorption spectra obtained from the fluorescence signal at the locations pointed to by the arrows in (b).

At each location on the tomographic slice, the absorption spectrum can now be evaluated. Four example spectra at locations pointed to by the arrows in Fig. 5, two obtained by absorption and two by fluorescence contrast, are shown in Fig. 5c-f. In pixels with high zinc concentration the spectra obtained by absorption and fluorescence are very similar (spectra $\mathrm{d}$ and $\mathrm{f}$ in Fig. 5). Where the zinc concentration is low, the absorption signal is dominated by low- $Z$ elements and the zinc spectrum is barely visible (Fig. 5c). In the fluorescence channel, however, a clean XANES spectrum is obtained with low noise as shown in Fig. 5e, illustrating the strength of the fluorescence detection in terms of signal-to-noise ratio.

Figure $5 \mathrm{e}-\mathrm{f}$ shows that zinc is found in different chemical environments at different locations in the root. These XANES spectra can now be analyzed, e.g., by comparing them to those of different zinc reference compounds. However, this task is not as straight forward as in the case of the catalysts studied in [53] as the zinc chemistry in plants is very complex, and further studies are required. This example shows that local absorption spectroscopy can be carried out with the help of XANES tomography even for relatively dilute systems. The zinc concentration in the plant, for example in bulk analysis, lies on average below 100 ppm.

\subsection{Coherent $X$-ray diffraction imaging with focused radiation}

In the last decade, coherent X-ray diffraction imaging (CXDI) techniques have emerged as an alternative X-ray microscopy method [93-100]. The method, also referred to as lensless imaging, relies on illuminating the sample by coherent light and recording a far-field diffraction pattern of it. From these data and with additional knowledge, e.g., the finite sample extension, the wave field behind the object can be reconstructed by numerical phase retrieval [101]. As no objective lens is involved in this imaging process, this method is not limited by any aberrations or a limited numerical aperture. Indeed, if a diffraction pattern can be recorded to large diffraction angles, in principle, spatial resolutions on the order of the wavelength are conceivable [102, 103].

Typically, however, the diffraction pattern of a nonperiodic object decays quickly to large diffraction angles, so that the main limitation of the technique is the coherent dose that can be applied to the sample. In many cases, radiation damage in the sample itself limits the resolution [105], but for radiation-hard samples, the limitation comes from the X-ray source and its coherent flux. One way to increase the coherent flux density on a small sample is to focus the $\mathrm{x}$ rays and thus increase the spatial resolution of the technique. We have pursued this latter scheme by illuminating small objects (diameter $<100 \mathrm{~nm}$ ) with the diffraction-limited focus in the scanning microscope described in Sec. 2.2. Details of the method and considerations about the coherence properties in the focused beam can be found in [106].

Here, we give an example to illustrate the method. With the scanning microscope at beamline ID13 of the ESRF, we focused hard X-rays $(E=15.25 \mathrm{keV})$ to slightly above $100 \times 200 \mathrm{~nm}^{2}$ and illuminated a cluster of four gold particles shown in Fig. 6a. This object contains about $7.5 \times 10^{6}$ gold atoms and thus it is a very weak scatterer. The diffraction pattern shown in Fig. $6 \mathrm{~b}$ was recorded in $20 \times 60 \mathrm{~s}$ (ESRF storage ring in 16-bunch mode) using a FReLoN CCD detector in $2 \times 2$ binning mode (effective pixel size $110 \mu \mathrm{m}$ ), located at a distance of $1850 \mathrm{~mm}$ behind the focus. By focusing the X-ray beam, the flux density on the sample was increased by roughly four orders of magnitude. Without focusing, the same diffraction pattern would have required exposure times of several months.

The object was reconstructed using the hybrid input-output [101] and the shrink-wrap method [107]. Figure $6 \mathrm{c}$ shows the averaged projection of the object obtained from 38 reconstructions with random initial phases. The contrast differs from that in the scanning electron micrograph in Fig. 6a, in that the reconstructed image measures the projected electron density of the object along the beam direction. Therefore, the nearly 


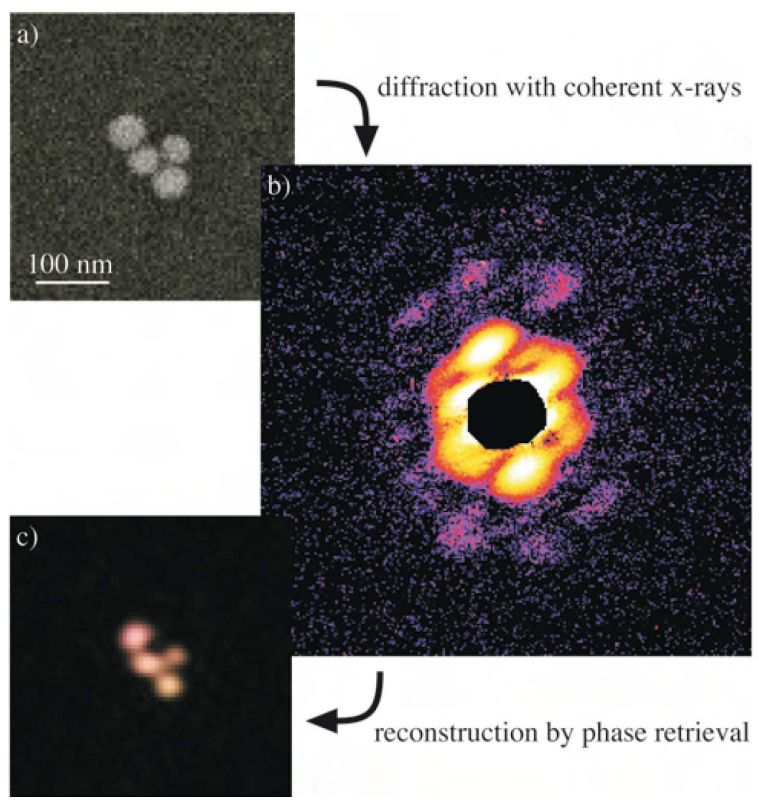

Fig. 6. (a) Cluster of four gold particles on a silicon nitride membrane used as test object for coherent X-ray diffraction imaging (scanning electron micrograph by I. Snigireva, ESRF). (b) Diffraction pattern recorded of the object in (a) by illuminating it with a coherent nanobeam at $E=15.25 \mathrm{keV}$. (c) Object as reconstructed from the diffraction pattern in (b).

spherical gold particles appear bright in their center and have less projected electron density and thus, appear darker towards their periphery. The spatial resolution obtained in this reconstruction can be measured using the phase-retrieval transfer function [97, 99]. In the current case, the latter drops below $10 \%$ at $8 \mathrm{~nm}$ half-periode indicating a spatial resolution in the range of $10 \mathrm{~nm}$. This example shows that with this technique spatial resolutions can be obtained that lie well beyond that of the scanning microscope.

In the present example, it was crucial that the object has a finite support smaller than the beam size. This requirement can be avoided by ptychographic imaging techniques [108, 109], where the sample is scanned in translation through a confined coherent illumination, such as the diffraction-limited focus of a scanning microscope. At each position of the scan, a far-field diffraction pattern is recorded. The object is then reconstructed from the set of diffraction patterns together with the knowledge of the positions where they were recorded. A first realization of this scheme is given in Ref. [110]. A significant advancement towards the practical application of this method was the joint reconstruction of sample and illumination at the same time [111], since the exact illumination is usually not known and not easily accessible otherwise. This technique has been implemented in our hard X-ray microscope to further increase the spatial resolution in transmission contrast [112].

\section{Conclusion and outlook}

Hard X-ray scanning microscopy takes full advantage of the strengths of hard X-rays. We have given two examples where it can deliver insight into the elemental composition and the chemistry of an element of interest. In combination with tomography, this information can also be obtained from the inside an object. In addition, structural information can be obtained by using X-ray diffraction as contrast, for example, in the small-angle scattering regime $[54,57]$.

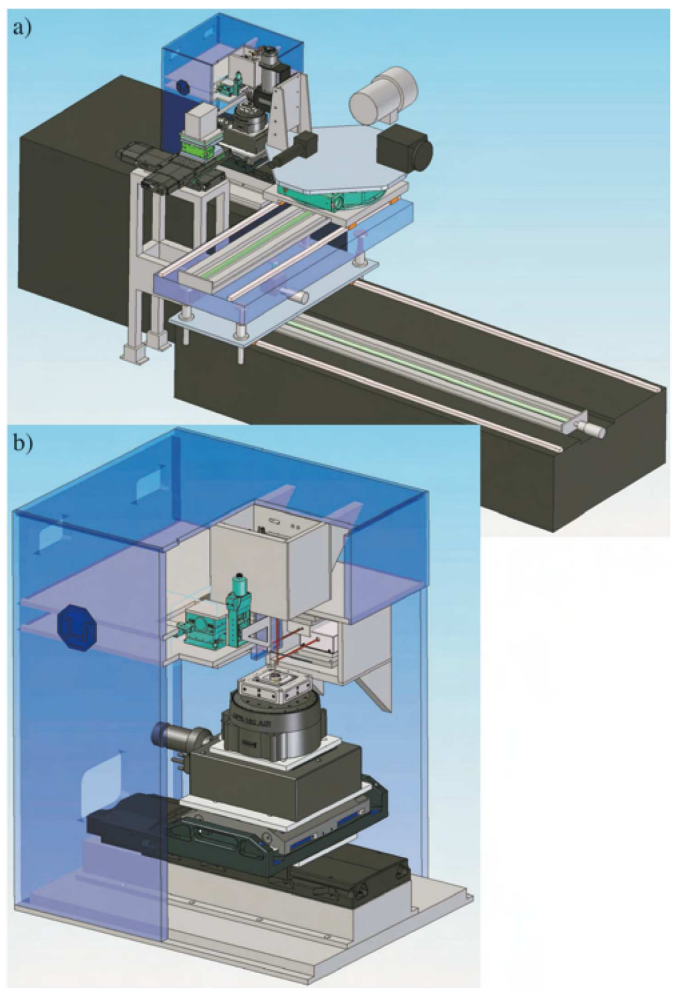

Fig. 7. (a) Design of the hard X-ray scanning microscope for the nanoprobe station at beamline P06 of the upcoming synchrotron radiation source PETRA III at DESY, Hamburg, Germany. The scanner unit is shown in more details in (b) and different detectors are placed on separate optical tables. The energy dispersive detector faces the sample perpendicularly to the optical axis. A high resolution X-ray camera, a light microscope, diodes, and diffraction cameras can be positioned behind the sample in SAXS and WAXS geometries.

While the spatial resolution depends on the scientific question at hand, there is the aim to reach a higher and higher spatial resolution. Currently, we achieve $100 \mathrm{~nm}$ spatial resolution on a routine basis with the hard X-ray scanning microscope at beamline ID13 at the ESRF. The goal is to improve the resolution down to well below $50 \mathrm{~nm}$ for routine operation.

Further improvements in spatial resolution down to the nanometer level are possible by combining focusing and scanning microscopy with coherent X-ray diffraction imaging techniques, as shown in the last example 
(Sect. 3.3.). Here, projections of the electron density of the sample can be obtained at a spatial resolution much higher than the focus size of the X-ray microscope. This type of microscopy holds great potential and will be pursued in the future.

Besides the development at the ESRF, a hard X-ray scanning microscope is planned for the new synchrotron radiation source PETRA III at DESY in Hamburg, Germany. This instrument is planned to provide a stable hard X-ray nanobeam in the range of $50 \mathrm{~nm}$ and below and will support fluorescence, absorption, and diffraction contrast. Figure 7 shows the overall design (a) and, in more detail, the scanning unit (b) of the instrument that is to be implemented at beamline P06 in the climate-controlled nanoprobe hutch at about $98 \mathrm{~m}$ from the source [113-115]. The X-rays are generated in a $2 \mathrm{~m}$ long spectroscopy undulator and are monochromatized by a Si (111) double crystal monochromator. The higher harmonics from the source are rejected with a pair of horizontally deflecting total reflection mirrors, and the lateral coherence length is adapted to the aperture of the nanofocusing optic by a parabolic refractive X-ray lens made of beryllium, located at $43.3 \mathrm{~m}$ from the source. The microscope is currently being built and is expected to be commissioned in early 2010 .

\section{Acknowledgments}

We would like to thank our collaborators M. Richwin and T.F. Günzler. This work is supported by the German Ministry of Education and Research (BMBF) under grant number 05KS7OD1 and by VI-203 of the Impulsund Vernetzungsfonds (IVF) of the Helmholtz-Society. Use of the Advanced Photon Source was supported by the U.S. Department of Energy, Office of Science, Office of Basic Energy Science, under Contract No. W-31-109-ENG-38.

\section{References}

[1] J.-D. Grunwaldt, S. Hannemann, C.G. Schroer, A. Baiker, J. Phys. Chem. B 110, 8674 (2006).

[2] B. Kimmerle, J.-D. Grunwaldt, A. Baiker, P. Glatzel, P. Boye, S. Stephan, C.G. Schroer, J. Phys. Chem. C 113, 3037 (2009).

[3] S. Bohic, A. Simionovici, A. Snigirev, R. Ortega, G. Devès, D. Heymann, C.G. Schroer, Appl. Phys. Lett. 78, 3544 (2001).

[4] A. Carmona, P. Cloetens, G. Deves, S. Bohic, R. Ortega, J. Analytical Atomic Spectrometry 23, 1083 (2008).

[5] R.H. Mathiesen, L. Arnberg, F. Mo, T. Weitkamp, A. Snigirev, Phys. Rev. Lett. 83, 5062 (1999).

[6] O. Castelnau, T. Chauveau, M. Drakopoulos, A. Snigirev, I. Snigireva, C. Schroer, T. Ungar, Mater. Sci. Forum 347-349, 297 (2000).

[7] W. Ludwig, S. Schmidt, E.M. Lauridsen, H.F. Poulsen, J. Appl. Crystallography 41, 302 (2008).
[8] G. Johnson, A. King, M.G. Honnicke, J. Marrow, W. Ludwig, J. Appl. Crystallography 41, 310 (2008).

[9] K.M. Kemner, W. Yun, Z. Cai, B. Lai, H.-R. Lee, J. Maser, D.G. Legnini, W. Rodrigues, J.D. Jastrow, R.M. Miller, S.T. Pratt, J. Synchrotron Rad. 6, 639 (1999).

[10] C.M. Hansel, S. Fendorf, S. Sutton, M. Newville, Environ. Sci. Technol. 35, 3863 (2001).

[11] M.C. Camerani, A. Pinzani, A.S. Somogyi, S. Simionovici, B.-M. Ansell, O. Steenari, Lindqvist, Environ. Sci. Technol. 36, 3165 (2002).

[12] D.H. McNear Jr., E. Peltier, J. Everhart, R.L. Chaney, S. Sutton, M. Newville, M. Rivers, D.L. Sparks, Environ. Sci. Technol. 39, 2210 (2005).

[13] R. Terzano, M. Spagnuolo, L. Medici, B. Vekemans, L. Vincze, K. Janssens, P. Ruggiero, Environ. Sci. Technol. 39, 6280 (2005).

[14] G. Silversmit, B. Vekemans, F.E. Brenker, S. Schmitz, M. Burghammer, C. Riekel, L. Vincze, Anal. Chem. 81, 6107 (2009).

[15] H.H. Solak, Y. Vladimirsky, F. Cerrina, B. Lai, W. Yun, Z. Cai, P. Illinski, D. Legnini, W. Rodrigues, J. Appl. Phys. 86, 884 (1999).

[16] T. Warwick, D. Attwood in: X-ray microscopy, AIP Conference Proceedings, Vol. 507, Ed. W. Meyer-Ilse, American Institute of Physics, Melville, New York 1999.

[17] in: Proceedings of the 8th International Conference on X-ray Microscopy, IPAP Conference Series, Vol. 7 , Ed. Y. Kagoshima, IPAP, Tokyo 2006.

[18] C.G. Schroer, P. Cloetens, M. Rivers, A. Snigirev, A. Takeuchi, W. Yun, MRS Bulletin 29, 157 (2004).

[19] A. Koch, C. Raven, P. Spanne, A. Snigirev, J. Opt. Soc. Am. A 15, 1940 (1998).

[20] M. Stampanoni, G. Borchert, P. Wyss, R. Abela, B. Patterson, S. Hunt, D. Vermeulen, P. Rüegsegger, Nucl. Instrum. Meth. A 491, 291 (2002).

[21] C. Raven, A. Snigirev, I. Snigireva, P. Spanne, S. Souvorov, V. Kohn, Appl. Phys. Lett. 69, 1826 (1996).

[22] M. Salome, F. Peyrin, P. Cloetens, C. Odet, A.M. Laval-Jeantet, J. Baruchel, P. Spanne, Med. Phys. 26, 2194 (1999).

[23] C.F. Martin, C. Josserond, L. Salvo, J.J. Blandin P. Cloetens, E. Boller, Scripta Mater. 42, 375 (2000).

[24] A. Snigirev, I. Snigireva, V. Kohn, S. Kuznetsov, I. Schelokov, Rev. Sci. Instrum. 66, 5486 (1995).

[25] T.E. Gureyev, C. Raven, A. Snigirev, I. Snigireva, S.W. Wilkins, J. Phys. D, Appl. Phys. 32, 563 (1999).

[26] P. Spanne, C. Raven, I. Snigireva, A. Snigirev, Phys. Med. Biol. 44, 741 (1999).

[27] P. Cloetens, W. Ludwig, J. Baruchel, D. Van Dyck, J. Van Landuyt, J.P. Guigay, M. Schlenker, Appl. Phys. Lett. 75, 2912 (1999).

[28] J.-Y. Buffière, E. Maire, P. Cloetens, G. Lormand, R. Fougères, Acta Mater. 47, 1613 (1999).

[29] T. Weitkamp, A. Diaz, C. David, F. Pfeiffer, M. Stampanoni, P. Cloetens, E. Ziegler, Opt. Express 13, 6296 (2005).

[30] F. Pfeiffer, O. Bunk, C. Grünzweig, C. David, M. Bech, G. Le Duc, A. Bravin, Phys. Med. Biol. 52, 6923 (2007). 
[31] S.A. McDonald, F. Marone, C. Hintermueller, G. Mikuljan, C. David, F. Pfeiffer, M. Stampanoni, J. Synchrotron Rad. 16, 562 (2009).

[32] S. Hannemann, J.-D. Grunwaldt, N. van Vegten, A. Baiker, P. Boye, C.G. Schroer, Catalysis Today 126, 54 (2006).

[33] J.-D. Grunwaldt, B. Kimmerle, S. Hannemann, A. Baiker, P. Boye, C.G. Schroer, J. Mat. Chem. 17, 2603 (2007)

[34] J.-D. Grunwaldt, B. Kimmerle, A. Baiker, P. Boye, C.G. Schroer, P. Glatzel, C.N. Borca, F. Beckmann, Catalysis Today 145, 267 (2009).

[35] B. Kimmerle, P. Haider, J.-D. Grunwaldt, A. Baiker, P. Boye, C.G. Schroer, Appl. Catal. A, Gen. 353, 36 (2009).

[36] C.G. Schroer, J.-D. Grunwaldt, Synchrotron Radiation News 22, 23 (2009).

[37] S. Lagomarsino, A. Cedola, P. Cloetens, S. Di, W. Fonzo, G. Jark, Soullié, C. Riekel, Appl. Phys. Lett. 71, 2557 (1997).

[38] R. Mokso, P. Cloetens, E. Maire, W. Ludwig, J.-Y. Buffière, Appl. Phys. Lett. 90, 144104 (2007).

[39] Y.S. Chu, J.M. Yi, F.D. Carlo, Q. Shen, W.-K. Lee, H.J. Wu, C.L. Wang, J.Y. Wang, C.J. Liu, C.H. Wang, S.R. Wu, C.C. Chien, Y. Hwu, A. Tkachuk, W. Yun, M. Feser, K.S. Liang, C.S. Yang, J.H. Je, G. Margaritondo, Appl. Phys. Lett. 92, 103119 (2008).

[40] B. Lengeler, C.G. Schroer, M. Richwin, J. Tümmler, M. Drakopoulos, A. Snigirev, I. Snigireva, Appl. Phys. Lett. 74, 3924 (1999).

[41] C.G. Schroer, J. Meyer, M. Kuhlmann, B. Benner, T.F. Günzler, B. Lengeler, C. Rau, T. Weitkamp, A. Snigirev, I. Snigireva, Appl. Phys. Lett. 81, 1527 (2002).

[42] C.G. Schroer, M. Kuhlmann, B. Lengeler, T.F. Günzler, O. Kurapova, B. Benner, C. Rau, A.S. Simionovici, A. Snigirev, I. Snigireva, in: Design and Microfabrication of Novel X-Ray Optics, Proceedings of the SPIE Vol. 4783, Ed. D.C. Mancini, SPIE, Bellingham 2002, p. 10

[43] G.E. Ice, B.C. Larson, MRS Bulletin 29, 170 (2004).

[44] O. Hignette, P. Cloetens, G. Rostaing, P. Bernard, C. Morawe, Rev. Sci. Instrum. 76, 063709 (2005).

[45] B. Golosio, A. Somogyi, A. Simionovici, P. Bleuet, J. Susini, L. Lamelle, Appl. Phys. Lett. 84, 2199 (2004).

[46] J.P. Hogan, R.A. Gonsalves, A.S. Krieger, IEEE Trans. Nucl. Sci. 38, 1721 (1991).

[47] L. Vincze, K. Janssens, B. Vekemans, F. Adams, in: Developments in X-ray Tomography II, Proceedings of the SPIE Vol. 37rg, Ed. U. Bonse, SPIE, Bellingham 1999 , p. 328.

[48] C.G. Schroer, Appl. Phys. Lett. 79, 1912 (2001).

[49] A. Brunetti, B. Golosio, Computer Physics Communications 141, 412 (2001).

[50] B. Golosio, A. Simionovici, A. Somogyi, L. Lamelle, M. Chukalina, A. Brunetti, J. Appl. Phys. 94, 145 (2003).

[51] B. Vekemans, L. Vincze, F.E. Brenker, F. Adams, J. Anal. At. Spectrom. 19, 1302 (2004).
[52] P.J. La, Rivière, D. Billmire, P. Vargas, M. Rivers, S.R. Sutton, Opt. Eng. 45, 077005 (2006).

[53] C.G. Schroer, M. Kuhlmann, T.F. Günzler, B. Lengeler, M. Richwin, B. Griesebock, D. Lützenkirchen-Hecht, R. Frahm, E. Ziegler, A. Mashayekhi, D. Haeffner, J.-D. Grunwaldt, A. Baiker, Appl. Phys. Lett. 82, 3360 (2003).

[54] C.G. Schroer, M. Kuhlmann, S.V. Roth, R. Gehrke, N. Stribeck, A. Almendarez-Camarillo, B. Lengeler, Appl. Phys. Lett. 88, 164102 (2006).

[55] J.M. Feldkamp, M. Kuhlmann, S.V. Roth, A. Timmann, R. Gehrke, I. Shakhverdova, P. Paufler, S.K. Filatov, R.S. Bubnova, C.G. Schroer, Phys. Status Solidi A 206, 1723 (2009).

[56] P. Bleuet, E. Welcomme, E. Dooryhée, J. Susini, J.-L. Hodeau, P. Walter, Nature Mater. 7, 468 (2008).

[57] M. Kuhlmann, J.M. Feldkamp, J. Patommel, S.V. Roth, A. Timmann, R. Gehrke, P. Müller-Buschbaum, C.G. Schroer, Langmuir Lett. 25, 7241 (2009).

[58] J.M. Feldkamp, C.G. Schroer, J. Patommel, B. Lengeler, T.F. Günzler, M. Schweitzer, C. Stenzel, M. Dieckmann, W.H. Schröder, Rev. Sci. Instrum. 78, 073702 (2007).

[59] W. Chao, B.D. Harteneck, J.A. Liddle, E.H. Anderson, D.T. Attwood, Nature 435, 1210 (2005).

[60] H. Mimura, H. Yumoto, S. Matsuyama, Y. Sano, K. Yamamura, Y. Mori, M. Yabashi, Y. Nishino, K. Tamasaku, T. Ishikawa, K. Yamauchi, Appl. Phys. Lett. 90, 051903 (2007).

[61] G.-C. Yin, Y.-F. Song, M.-T. Tang, F.-R. Chen, K.S. Liang, F.W. Duewer, M. Feser, W. Yun, H.-P.D. Shieh, Appl. Phys. Lett. 89, 221122 (2006).

[62] H.C. Kang, H. Yan, R.P. Winarski, M.V. Holt, J. Maser, C. Liu, R. Conley, S. Vogt, A.T. Macrander, G.B. Stephenson, Appl. Phys. Lett. 92, 221114 (2008).

[63] R. Conley, C. Liu, J. Qian, C.M. Kewish, A.T. Macrander, H. Yan, H.C. Kang, J. Maser, G.B. Stephenson, Rev. Sci. Instrum. 79, 053104 (2008).

[64] A. Jarre, C. Fuhse, C. Ollinger, J. Seeger, R. Tucoulou, T. Salditt, Phys. Rev. Lett. 94, 074801 (2005).

[65] C.G. Schroer, O. Kurapova, J. Patommel, P. Boye, J. Feldkamp, B. Lengeler, M. Burghammer, C. Riekel, L. Vincze, A. van der Hart, M. Küchler, Appl. Phys. Lett. 87, 124103 (2005).

[66] C. Bergemann, H. Keymeulen, J.F. van der Veen, Phys. Rev. Lett. 91, 204801 (2003).

[67] C.G. Schroer, B. Lengeler, Phys. Rev. Lett. 94 054802 (2005).

[68] C.G. Schroer, Phys. Rev. B 74, 033405 (2006).

[69] H. Yan, J. Maser, A. Macrander, Q. Shen, S. Vogt, G.B. Stephenson, H.C. Kang, Phys. Rev. B 76, 115438 (2007).

[70] A. Snigirev, V. Kohn, I. Snigireva, B. Lengeler, Nature 384, 49 (1996).

[71] B. Lengeler, J. Tümmler, A. Snigirev, I. Snigireva, C. Raven, J. Appl. Phys. 84, 5855 (1998). 
[72] Y. Kohmura, M. Awaji, Y. Suzuki, T. Ishikawa, Y.I. Dudchik, N.N. Kolchewsky, F.F. Komarow, Rev. Sci. Instrum. 70, 4161 (1999).

[73] J.T. Cremer, M.A. Piestrup, H.R. Beguiristain, C.K. Gary, R.H. Pantell, R. Tatchyn, Rev. Sci. Instrum. 70, 3545 (1999).

[74] M.A. Piestrup, J.T. Cremer, H.R. Beguiristain, C.K. Gary, R.H. Pantell, Rev. Sci. Instrum. 71, 4375 (2000).

[75] B. Cederström, R.N. Cahn, M. Danielsson, M. Lundqvist, D.R. Nygren, Nature 404, 951 (2000).

[76] V. Aristov, M. Grigoriev, S. Kuznetsov, L. Shabelnikov, V. Yunkin, T. Weitkamp, C. Rau, I. Snigireva, A. Snigirev, M. Hoffmann, E. Voges, Appl. Phys. Lett. 77, 4058 (2000).

[77] E.M. Dufresne, D.A. Arms, R. Clarke, N.R. Pereira, S.B. Dierker, D. Foster, Appl. Phys. Lett. 79, 4085 (2001).

[78] H.R. Beguiristain, J.T. Cremer, M.A. Piestrup, C.K. Gary, R.H. Pantell, Opt. Lett. 27, 778 (2002).

[79] K. Evans-Lutterodt, J.M. Ablett, A. Stein, C.-C. Kao, D.M. Tennant, F. Klemens, A. Taylor, C. Jacobsen, P.L. Gammel, H. Huggins, S. Ustin, G. Bogart, L. Ocula, Optics Express 11, 919 (2003).

[80] B. Lengeler, C.G. Schroer, M. Kuhlmann, B. Benner, T.F. Günzler, O. Kurapova, F. Zontone, A. Snigirev, I. Snigireva, J. Phys. D, Appl. Phys. 38, A218 (2005).

[81] B. Nöhammer, J. Hoszowska, A.K. Freund, C. David, J. Synchrotron Rad. 10, 168 (2003).

[82] B. Lengeler, C. Schroer, J. Tümmler, B. Benner, M. Richwin, A. Snigirev, I. Snigireva, M. Drakopoulos, J. Synchrotron Rad. 6, 1153 (1999).

[83] C.G. Schroer, B. Benner, T.F. Günzler, M. Kuhlmann, C. Zimprich, B. Lengeler, C. Rau, T. Weitkamp, A. Snigirev, I. Snigireva, J. Appenzeller, Rev. Sci. Instrum. 73, 1640 (2002).

[84] C.G. Schroer, B. Lengeler, B. Benner, T.F. Günzler, M. Kuhlmann, A.S. Simionovici, S. Bohic, M. Drakopoulos, A. Snigirev, I. Snigireva, W.H. Schröder, Proc. SPIE 4499, 52 (2001).

[85] S.V. Roth, R. Döhrmann, M. Dommach, M. Kuhlmann, I. Kröger, R. Gehrke, H. Walter, C. Schroer, B. Lengeler, P. Müller-Buschbaum, Rev. Sci. Instrum. 77, 085106 (2006).

[86] C.G. Schroer, M. Kuhlmann, U.T. Hunger, T.F. Günzler, O. Kurapova, S. Feste, F. Frehse, B. Lengeler, M. Drakopoulos, A. Somogyi, A.S. Simionovici, A. Snigirev, I. Snigireva, C. Schug, W.H. Schröder, Appl. Phys. Lett. 82, 1485 (2003).

[87] A.C. Kak, M. Slaney, Principles of Computerized Tomographic Imaging, IEEE Press, New York 1988.

[88] A.S. Simionovici, M. Chukalina, C. Schroer, M. Drakopoulos, A. Snigirev, I. Snigireva, B. Lengeler, K. Janssens, F. Adams, IEEE Trans. Nucl. Sci. 47, 2736 (2000)

[89] C.G. Schroer, J. Tümmler, T.F. Günzler, B. Lengeler, W.H. Schröder, A.J. Kuhn, A.S. Simionovici, A. Snigirev, I. Snigireva, Proc. SPIE 4142, 287 (2000).

[90] M. Richwin, R. Zaeper, D. Lützenkirchen-Hecht, R. Frahm, J. Synchrotron Rad. 8, 354 (2001).
[91] R. Frahm, J. Stötzel, D. Lützenkirchen-Hecht, Synchrotron Radiation News 22, 6 (2009).

[92] C.G. Schroer, M. Kuhlmann, T.F. Günzler, B. Lengeler, M. Richwin, B. Griesebock, D. Lützenkirchen-Hecht, R. Frahm, A. Mashayekhi, D. Haeffner, E. Ziegler, J.-D. Grunwaldt, A. Baiker, Physica Scripta T 115, 1026 (2005).

[] J. Miao, P. Charalambous, J. Kirz, D. Sayre, Nature 400, 342 (1999).

[94] I.K. Robinson, I.A. Vartanyants, G.J. Williams, M.A. Pfeifer, J.A. Pitney, Phys. Rev. Lett. 87, 195505 (2001).

[95] J. Miao, T. Ishikawa, B. Johnson, E.H. Anderson, B. Lai, K.O. Hodgson, Phys. Rev. Lett. 89, 088303 (2002).

[96] G.J. Williams, M.A. Pfeifer, I.A. Vartanyants, I.K. Robinson, Phys. Rev. Lett. 90, 175501 (2003).

[97] D. Shapiro, P. Thibault, T. Beetz, V. Elser, M. Howells, C. Jacobsen, J. Kirz, E. Lima, H. Miao, A. Neiman, D. Sayre, Proc. Natl. Acad. Sci. USA 102, $15343(2005)$.

[98] J. Miao, Y. Nishino, Y. Kohmura, B. Johnson, C. Song, S.H. Risbud, T. Ishikawa, Phys. Rev. Lett. 95, 085503 (2005).

[99] H.N. Chapman, A. Barty, S. Marchesini, A. Noy, S.P. Hau-Riege, C. Cui, M.R. Howells, R. Rosen, H. He, J.C.H. Spence, U. Weierstall, T. Beetz, C. Jacobsen, D. Shapiro, J. Opt. Soc. Am. A 23, 1179 (2006).

[100] M.A. Pfeifer, G.J. Williams, I.A. Vartanyants, R. Harder, I.K. Robinson, Nature 442, 63 (2006).

[101] J.R. Fienup, Appl. Opt. 21, 2758 (1982).

[102] R. Neutze, R. Wouts, D. van der Spoel, E. Weckert, J. Hajdu, Nature 406, 752 (2000).

[103] J. Miao, K.O. Hodgson, D. Sayre, Proc. Natl. Acad. Sci. USA 98, 6641 (2001).

[104] D. Starodub, P. Rez, G. Hembree, M.R. Howells, D.A. Shapiro, H.N. Chapman, P. Fromme, K. Schmidt, U. Weierstall, R.B. Doak, J.C.H. Spence, J. Synchrotron Rad. 15, 62 (2007).

[105] M.R. Howells, T. Beetz, H.N. Chapman, C. Cui, J.M. Holton, C.J. Jacobsen, J. Kirz, E. Lima, S. Marchesini, H. Miao, D. Sayre, D.A. Shapiro, J.C.H. Spence, D. Starodub, J. Electron Spect. 170, 4 (2009).

[106] C.G. Schroer, P. Boye, J. Feldkamp, J. Patommel, A. Schropp, A. Schwab, S. Stephan, M. Burghammer, S. Schöder, C. Riekel, Phys. Rev. Lett. 101, 090801 (2008).

[107] S. Marchesini, H. He, H.N. Chapman, S.P. Hau-Riege, A. Noy, M.R. Howells, U. Weierstall, J.C.H. Spence, Phys. Rev. B 68, 140101(R) (2003).

[108] J.M. Rodenburg, H.M.L. Faulkner, Appl. Phys. Lett. 85, 4795 (2004).

[109] H.M.L. Faulkner, J.M. Rodenburg, Phys. Rev. Lett. 93, 023903 (2004).

[110] J.M. Rodenburg, A.C. Hurst, A.G. Cullis, B.R. Dobsen, F. Pfeiffer, O. Bunk, C. David, K. Jefimovs, I. Johnson, Phys. Rev. Lett. 98, 034801 (2007).

[111] P. Thibault, M. Dierolf, A. Menzel, O. Bunk, C. David, F. Pfeiffer, Science 321, 379 (2008). 
[112] A. Schropp, P. Boye, J.M. Feldkamp, R. Hoppe, J. Patommel, D. Samberg, S. Stephan, K. Giewekemeyer, R.N. Wilke, T. Salditt, J. Gulden, A.P. Mancuso, I.A. Vartanyants, E. Weckert, S. Schöder, M. Burghammer, C.G. Schroer, Appl. Phys. Lett. 96, 091102 (2010).

[113] K. Balewski, W. Brefeld, W. Decking, H. Franz, R. Röhlsberger, E. Weckert, PETRA III: A Low Emittance Synchrotron Radiation Source, DESY 2004-035, DESY, Hamburg 2004.
[114] G. Falkenberg, N. Reimers, C.G. Schroer, in: HASYLAB Jahresbericht, DESY, Hamburg 2007, p. 185.

[115] C.G. Schroer, P. Boye, J.M. Feldkamp, J. Patommel, D. Samberg, A. Schropp, A. Schwab, S. Stephan, G. Falkenberg, G. Wellenreuther, N. Reimers, Nucl. Instrum Methods A (2009), Doi: 10.1016/ j.nima.2009.10.094. 